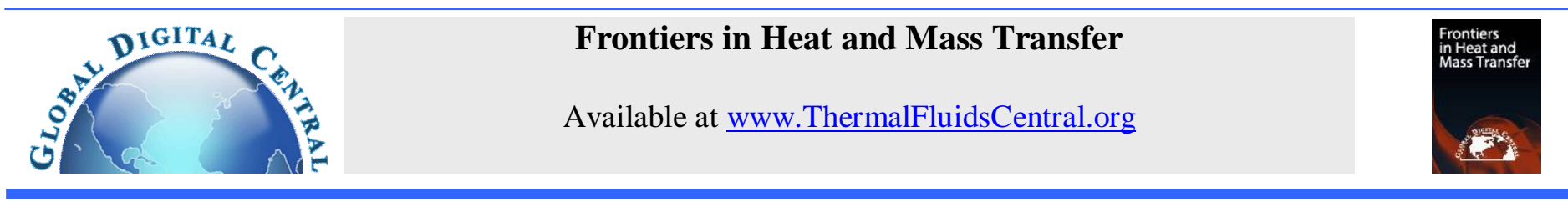

\title{
VISCOUS DISSIPATION EFFECT ON TRANSIENT ALIGNED MAGNETIC FREE CONVECTIVE FLOW PAST AN INCLINED MOVING PLATE
}

\author{
G. Dharmaiah ${ }^{\mathrm{a}^{*}}$, Ali J. Chamkha ${ }^{\mathrm{b}}$, N. Vedavathi ${ }^{\mathrm{c}}$, K.S. Balamurugan ${ }^{\mathrm{d}}$ \\ ${ }^{a}$ Department of Mathematics, Narasaraopeta Engineering College, Yellamanda, Narasaraopet, Andhra Pradesh, 522601, India. \\ ${ }^{b}$ Mechanical Engineering Department, Prince Sultan Endowment for Energy and Environment, Prince Mohammad Bin Fahd University, \\ Al-Khobar 31952, Saudi Arabia. \\ ${ }^{c}$ Department of Mathematics, Koneru Lakshmaiah Education Foundation, Vaddeswaram, Guntur, Andhra Pradesh, 522502, India. \\ ${ }^{d}$ Department of Mathematics, RVR \& JC College of Engineering, Chowdavaram, Guntur, Andhra Pradesh, 522019, India.
}

\begin{abstract}
The present analysis is focused on free convective heat and mass transfer characteristics of magneto flow through a moving inclined plate under the influence of Aligned magnetic, viscous dissipation and thermal radiation. A uniform magnetic field is applied perpendicular to the plate. The governing non-dimensional linear partial differential equations are solved by using perturbation technique. Graphical results for the velocity, temperature and concentration distributions within the boundary layer for several physical parameters and tabulated results for the Skin-friction, the Nusselt number and the Sherwood number are displayed and discussed. The effect of increasing values of the viscous dissipation parameter or the Eckert number is to enhance the velocity and temperature fields. The current study is well supported by the verification of a previous result.
\end{abstract}

Keywords: Chemical Reaction, Radiation, Viscous dissipation, Inclined plate, Aligned Magnetic.

\section{INTRODUCTION}

A magnetohydrodynamic flow refers to the flow of electrically conducting fluids, such as plasmas or ionized gases under a magnetic field. The effect of the magnetic field on the flow of the fluid can be derived using Reynolds's transport theorem and Maxwell's equations. Magnetohydrodynamics is currently undergoing a period of great enlargement and differentiation of subject matter. The interest in these new problems generates from their importance in liquid metals, electrolytes and ionized gases. This concept has been surveyed and reported in the research paper by Chandran et al. (1998). Convection in porous media can be applied to underground coal gasification, ground water hydrology, iron blast furnaces, wall cooled catalytic reactors, cooling of nuclear reactors, solar power collectors, energy efficient drying processes, cooling of electronic equipments and natural convection in earth's crust. Heat and mass transfer from different geometries embedded in porous media has many engineering and geophysical applications such as drying of porous solids, thermal insulations, cooling of nuclear reactors, crude oil extraction, underground energy transport, etc. The hydromagnetic convection with heat and mass transfer in porous medium has been studied due to its importance in the design of MHD generators and accelerators in geophysics, in design of underground water energy storage system, soilsciences, astrophysics, nuclear power reactors and so on. The concept of porous medium with heat and mass transfer has been studied and presented in the research paper by Reddy (2014).

Thermal radiation impacts might play a major role in controlling heat transfer processes in polymer processing industry. High temperature plasma, cooling of nuclear reactors and power generation systems. The radiative flows of an electrically conducting fluid with high temperature in the presence of magnetic field are experienced in electrical power era, space vehicle reentry, atomic designing applications and other modern zones. Owing to these applications, the present work deals with a problem of such kind. MHD boundary layer flow over an exponentially stretching permeable surface with thermal radiation is studied by Mukhopadhyaya et al. (2014).

The flow and heat transfer analysis in boundary layer flow over an exponentially stretching sheet with combined effects of suction/blowing and thermal radiation are investigated by Pramanik (2014). Pushpalatha et al. (2016) investigated the effects of thermal diffusion and radiation with convective boundary conditions. Effects of thermal radiation and porosity on MHD mixed convection flow in a vertical channel using homotopy analysis method were also carried out by Srinivas and Muthuraj (2010). Raptis et al. (2003) studied the effects of radiation in an optically thin gray gas flowing past a vertical infinite plate in the presence of a magnetic field. Raju et al. (2014) presented an analytical study of MHD free convictive, dissipative boundary layer flow past a porous vertical surface in the presence of thermal radiation, chemical reaction and constant suction. 
The effect of viscous dissipation plays an important role in natural convection in different devices which are subjected to large variations of gravitational force or which operate at high speeds. The energy dissipated due to motion of the fluid are retardation due to the application of magnetic field into the system, has a heating/cooling effect on the surface which result in significant heat transfer to the fluid in the boundary layer region. Viscous dissipation characterizes the degeneration of mechanical energy into the thermal energy. Such phenomenon transpires in all the flow systems. However, for different flow configurations, the characteristics of viscous dissipation are often neglected. It is meaningful just for the systems having larger velocity and velocity gradients respectively. It is for this reason that the viscous dissipation is introduced in the present study. Viscous dissipation is of interest for many applications: significant temperature rises were observed in polymer processing flows such as injection modeling or extrusion at high rates. Medikar et al. (2016) analyzed MHD stagnation point flow of a over a non linear stretching sheet with viscous dissipation. The Influence of viscous dissipation on the MHD natural convective flow over an oscillating vertical plate is analyzed by Reddy et al. (2017). Steady, incompressible, laminar flow of an electrically conducting Casson fluid over a melting surface on the upper horizontal paraboloid of revolution in the presence of viscous dissipation is studied by Ajayi et al. (2017) . Recently, steady magnetohydrodynamic (MHD) flow in the stretching surface with viscous dissipation effect examined by Tamoor et al. (2017). The study of chemical reaction with heat transfer in porous medium has important engineering applications e.g., tabular reactors, oxidation of solid materials and synthesis of ceramic materials reported by Shehzad et al. (2013). However, studies (Sandeep and Jagadeesh (2016), Rawi et al. (2016) and Amit (2017)) which focused on non-Newtonian flows along inclined surfaces demonstrate significant influence of inclination on MHD thermo fluid dynamic characteristics.

Motivated by the above studies, in this manuscript an attempt is made to investigate the effect of viscous dissipation on a radiative, mixed convection aligned MHD flow of a viscous, incompressible, electrically conducting and Newtonian fluid on a moving inclined porous plate in the presence of aligned magnetic field. The objective of this article is the effects of mixed convection with thermal radiation, viscous dissipation and chemical reaction on MHD flow of viscous, incompressible and electrically conducting fluid on a moving inclined heated porous plate is analyzed. The nonlinear coupled partial differential equations are solved analytically by employing perturbation technique. Graphical results for the velocity, temperature and concentration distributions within the boundary layer for several physical parameters and tabulated results for the skin-friction, the Nusselt number and the Sherwood number are presented and discussed.

\section{ANALYSIS OF THE FLOW OF THE PROBLEM}

Consider unsteady two-dimensional flow of an incompressible, viscous, electrically conducting and heat-absorbing fluid past a semi-infinite inclined permeable plate embedded in a uniform porous medium which is subject to a uniform transverse magnetic field in the presence of thermal and buoyancy effects in the direction of $y^{*}$ - axis. The transversely applied magnetic field and magnetic Reynolds number are assumed to be very small so that Hall Effect is negligible. It is assumed that there is no applied voltage which implies the absence of electric field. The wall is maintained at constant temperature $\mathrm{T}_{\mathrm{w}}$ and concentration $\mathrm{C}_{\mathrm{w}}$, higher than the ambient temperature $T_{\infty}$ and the concentration $C_{\infty}$, respectively. It is assumed that the porous medium is homogeneous and present everywhere in local thermodynamic equilibrium. Rest of properties of fluid and the porous medium are assumed to be constant. The concentration of the diffusing species in the binary mixture is assumed to be very small in comparison with the other chemical species which are present, and hence the Soret and Dufour effects are negligible. Further due to the semi-infinite plane surface assumption, the flow variables are functions of normal distance $\mathrm{y}^{*}$ and $\mathrm{t}^{*}$ only.

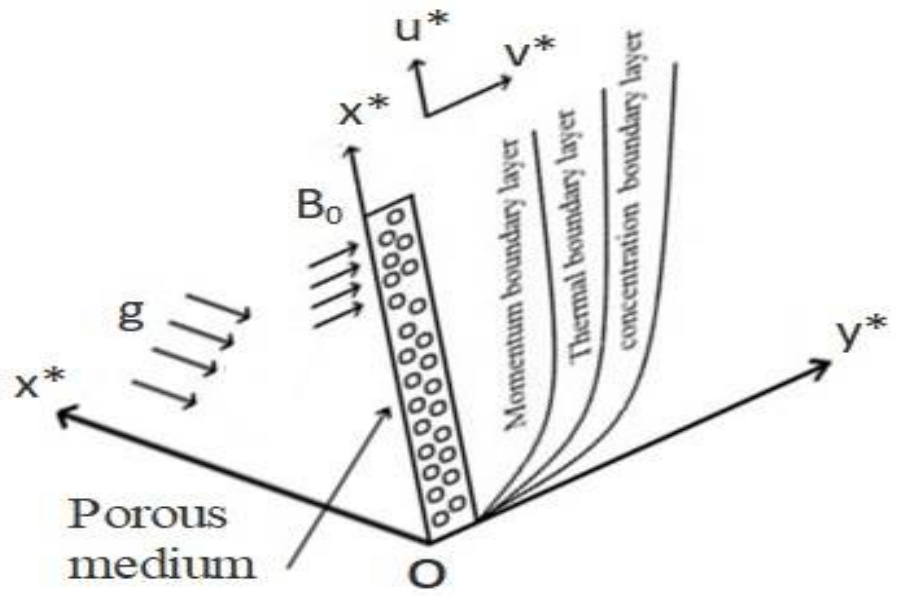

Fig. 1 Geometry of the problem

In the above assumptions and the usual Boussinesq's approximation, the governing boundary layer equations as follows:

Continuity Equation:

$\frac{\partial v^{*}}{\partial y^{*}}=0$

Momentum Equation:

$\frac{\partial u^{*}}{\partial t^{*}}+v^{*} \frac{\partial u^{*}}{\partial y^{*}}=-\frac{1}{\rho} \frac{\partial p^{*}}{\partial x^{*}}+v \frac{\partial^{2} u^{*}}{\partial y^{*^{2}}}-\frac{\sigma B_{0}^{2}}{\rho} u^{*} \sin ^{2} \xi+$

$g \cos \alpha \beta\left[T^{*}-T_{\infty}^{*}\right]+g \cos \alpha \beta^{*}\left[C^{*}-C_{\infty}^{*}\right]-\frac{v}{K^{*}} u^{*}$

Energy Equation:

$\frac{\partial T^{*}}{\partial t^{*}}+v^{*} \frac{\partial T^{*}}{\partial y^{*}}=\alpha \frac{\partial^{2} T}{\partial y^{*^{2}}}-\frac{1}{\rho C_{p}} \frac{\partial q_{r}}{\partial y^{*}}+\frac{v}{C p}\left[\frac{\partial u^{*}}{\partial y^{*}}\right]^{2}$

Mass Diffusion Equation:

$\frac{\partial C^{*}}{\partial t^{*}}+v^{*} \frac{\partial C^{*}}{\partial y^{*}}=D \frac{\partial^{2} C^{*}}{\partial y^{*^{2}}}-K_{r}^{*}\left(C^{*}-C_{\infty}^{*}\right)$

Where $x^{*}$ and $y^{*}$ are, the dimensional distances along to the plate, $u^{*}$ and $v^{*}$ are the components of dimensional velocities along $x^{*}$ and $y^{*}$ directions. $\mathrm{G}$ is the gravitational acceleration, $T^{*}$ is the dimensional temperature of the fluid near the plate, $T_{\infty}$ is the stream dimensional temperature, $C^{*}$ is the dimensional concentration, $C_{\infty}$ is the stream dimensional concentration, $\beta$ and $\beta^{*}$ are the thermal and concentration expansion coefficients, respectively. $p^{*}$ is the pressure, $C_{p}$ is the specific heat of constant pressure, $B_{0}$ is the magnetic field coefficient, $\mu$ is the viscosity of the fluid, $q_{r}^{*}$ is the radiative heat flux, $\rho$ is the density, $\mathrm{K}$ is the thermal conductivity, $\sigma$ is the density magnetic permeability of the fluid, $\mathrm{D}$ is the molecular diffusivity,

$v=\frac{\mu}{\rho}$ is the kinematic viscosity. 
The local radiant absorption for the case of an optically thin gray gas is expressed as $q_{r}=-\frac{4 \sigma^{*}}{3 k^{*}} \frac{\partial T^{4}}{\partial y^{*}} ; \frac{\partial q_{r}}{\partial y^{*}}=-\frac{16 \sigma^{*} T_{\infty}^{3}}{3 k^{*}} \frac{\partial^{2} T}{\partial y^{2}}$

Where $\sigma^{*}$ and $k^{*}$ are the Stefan-Boltzmann constant and the Mean absorption coefficient, respectively. We assume that the temperature differences within the flow are sufficiently small so that $T^{4}$ can be expressed as a linear function of $T$ after using Taylor's series to expand $T^{4}$ about the free stream temperature $T_{\infty}$ and neglecting higher-order terms. This results in the following approximation:

$T^{4} \cong 4 T_{\infty}^{3} T-3 T_{\infty}^{4}$

The fourth and fifth terms of RHS of the Eq.(2) denote the thermal and concentration buoyancy effects on inclined plate, respectively. The second and third term on the RHS of the Eq.(3) denote the inclusion of the effect of thermal radiation and viscous dissipation respectively.

$u^{*}=u_{p}^{*}, T^{*}=T_{w}^{*}+\varepsilon\left(T_{w}^{*}-T_{\infty}^{*}\right) e^{n^{*} t^{*}}, C^{*}=C_{w}^{*}+\varepsilon\left(C_{w}^{*}-C_{\infty}^{*}\right) e^{n^{*} t^{*}}$ at $y^{*}=0$

$u^{*}=U_{\infty}^{*}=U_{0}\left(1+\varepsilon e^{n^{*} t^{*}}\right), \quad T^{*} \rightarrow T_{\infty}^{*}, \quad C^{*} \rightarrow C_{\infty}^{*}$ as $y^{*} \rightarrow \infty$

Where $U_{p}, C_{w}$ and $T_{w}$ are the wall dimensional velocity, concentration and temperature, respectively. $U_{\infty}^{*}, C_{\infty}^{*}$ and $T_{\infty}^{*}$ are the free stream dimensional velocity, concentration and temperature, respectively. $U_{0}$ and $n^{*}$ are constants. It is clear from Eq. (1) that the suction velocity at the plate surface is a function of time only. Assuming that it takes the following exponential form:

$v^{*}=-V_{0}\left(1+\varepsilon A e^{n^{*} t^{*}}\right)$

Where A is a real positive constant, $\varepsilon$ and $\varepsilon A$ are small less than unity, and $V_{0}$ is a scale of suction velocity which has non-zero positive constant. Outside the boundary layer, Eq.(2) gives

$-\frac{1}{\rho} \frac{d p^{*}}{d x^{*}}=\frac{d U_{\infty}^{*}}{d t^{*}}+\frac{\sigma}{\rho} B_{0}^{2} U_{\infty}^{*}+\frac{v}{K^{*}} U_{\infty}^{*}$

Introducing the non-dimensional quantities

$$
\begin{aligned}
& u=\frac{u^{*}}{U_{0}}, v=\frac{v^{*}}{V_{0}}, y=\frac{v_{0} y^{*}}{v}, U_{\infty}=\frac{U_{\infty}^{*}}{U_{0}}, t=\frac{V_{0}^{2} t^{*}}{v}, \theta=\frac{T^{*}-T_{\infty}^{*}}{T_{w}^{*}-T_{\infty}^{*}}, \\
& C=\frac{C^{*}-C_{\infty}^{*}}{C_{w}^{*}-C_{\infty}^{*}}, n=\frac{n^{*} v}{V_{0}^{2}}, U_{p}=\frac{u_{p}^{*}}{U_{0}}, G r=\frac{g v\left(T_{w}-T_{\infty}\right) \beta}{U_{0} V_{0}^{2}}, \\
& G m=\frac{g v\left(C_{w}-C_{\infty}\right) \beta^{*}}{U_{0} V_{0}^{2}}, M=\frac{\sigma v B_{0}^{2}}{\rho V_{0}^{2}}, \operatorname{Pr}=\frac{v \rho C_{p}}{k}=\frac{v}{\alpha}, \\
& S c=\frac{v}{D}, K r=\frac{K_{1}^{*} v}{V_{0}^{2}}, K=\frac{V_{0}^{2} K^{*}}{V^{2}}, E c=\frac{U_{0}^{2}}{C p\left(T_{w}^{*}-T_{\infty}^{*}\right)}, \\
& R^{2}=\frac{\left(T_{w}^{*}-T_{\infty}^{*}\right) \alpha_{1}^{2}}{\rho C_{p} k U_{0}^{2}}, G r_{i}=G r \cos \alpha, G m_{i}=G m \cos \alpha, \\
& M a=M \sin ^{2} \xi .
\end{aligned}
$$

In the view of the above non-dimensional variables, the basic field of
Eqs. (2) - (4) can be expressed in non-dimensional form as $\frac{\partial u}{\partial t}-\left(1+\varepsilon A e^{n t}\right) \frac{\partial u}{\partial y}=\frac{d U_{\infty}}{d t}+\frac{\partial^{2} u}{\partial y^{2}}+G r_{i} \theta+G m_{i} C+N\left(U_{\infty}-u\right)$

$\frac{\partial \theta}{\partial t}-\left(1+\varepsilon A e^{n t}\right) \frac{\partial \theta}{\partial y}=\frac{1}{\operatorname{Pr}} \frac{\partial^{2} \theta}{\partial y^{2}}-\frac{1}{\operatorname{Pr}} R^{2}+E c\left(\frac{\partial u}{\partial y}\right)^{2}$

$\frac{\partial C}{\partial t}-\left(1+\varepsilon A e^{n t}\right) \frac{\partial C}{\partial y}=\frac{1}{S c} \frac{\partial^{2} C}{\partial y^{2}}-K r C$

Where, $N=\left(M a+\frac{1}{K}\right)$ and $\mathrm{Gr}, \mathrm{Gm}, \mathrm{Pr}, \mathrm{R}, \mathrm{Ec}, \mathrm{Sc}, \mathrm{Kr}, \alpha$ and $\xi$ are the thermal Grashof number, Solutal Grashof number, Prandtl number, radiation parameter, viscous dissipation parameter, Schmidt number, Chemical reaction parameter, inclined angle and Aligned magnetic parameter respectively.

The corresponding boundary conditions (5) and (6) in dimensionless form are

$$
\begin{aligned}
& u=U_{p}, \quad \theta=1+\varepsilon e^{n t}, \quad C=1+\varepsilon e^{n t}, \quad \text { at } \quad y=0 \\
& u \rightarrow U_{\infty}=\left(1+\varepsilon e^{n t}\right), \quad \theta \rightarrow 0, \quad C \rightarrow 0 \quad \text { as } \quad y \rightarrow \infty
\end{aligned}
$$

\section{METHOD OF SOLUTION}

When the amplitude of oscillations $(\varepsilon<<1)$ is very small. We can assume the solutions of flow velocity $\mathrm{u}$, temperature $\theta$ and concentration $\mathrm{C}$ in the neighborhood of the plate is:

$\mathrm{u}=\mathrm{f}_{0}(\mathrm{y})+\varepsilon \mathrm{e}^{\mathrm{nt}} \mathrm{f}_{1}(\mathrm{y})+\mathrm{O}\left(\varepsilon^{2}\right)+\cdots \cdots \cdot$
$\theta=\mathrm{g}_{0}(\mathrm{y})+\varepsilon \mathrm{e}^{\mathrm{nt}} \mathrm{g}_{1}(\mathrm{y})+\mathrm{O}\left(\varepsilon^{2}\right)+\cdots \cdots \cdot$
$\mathrm{C}=\mathrm{h}_{0}(\mathrm{y})+\varepsilon \mathrm{e}^{\mathrm{nt}} \mathrm{h}_{1}(\mathrm{y})+\mathrm{O}\left(\varepsilon^{2}\right)+\cdots \cdots \cdots$

Substituting (15)-(17) into Eqs.(10)-(12) and equating the harmonic and nonharmonic terms, neglecting the higher order $\mathrm{O}\left(\varepsilon^{2}\right)$, and simplifying to get the following pairs of equations for $f_{0}, g_{0}, h_{0}$ and $f_{1}, g_{1}$, $\mathrm{h}_{1}$.

$f_{0}^{\prime \prime}+f_{0}^{\prime}-N f_{0}=-G r_{i} g_{0}-G m_{i} h_{0}-N$

$f_{1}^{\prime \prime}+f_{1}^{\prime}-(N+n) f_{1}=-A f_{0}^{\prime}-G r_{i} g_{1}-G m_{i} h_{1}-(n+N)$

$g_{0}^{\prime \prime}+\operatorname{Pr} g_{0}^{\prime}-R^{2} g_{0}=-\operatorname{Pr} E c\left(f_{0}^{\prime}\right)^{2}$

$g_{1}^{\prime \prime}+\operatorname{Pr} g_{1}^{\prime}-\left(R^{2}+n \operatorname{Pr}\right) g_{1}=-\operatorname{Pr} A g_{0}^{\prime}-2 \operatorname{Pr} E c f_{0}^{\prime} f_{1}^{\prime}$

$h_{0}^{\prime \prime}+S c h_{0}^{\prime}-S c K r h_{0}=0$

$h_{1}^{\prime \prime}+h_{1}^{\prime} S c-(K r+n) S c h_{1}=-A h_{0}^{\prime} S c$

Where the prime denotes ordinary differentiation with respect to $y$.

The corresponding boundary conditions can be written as

$f_{0}=U_{p}, f_{1}=0, g_{0}=1, g_{1}=1, h_{0}=1, h_{1}=1$, at $\quad y=0$

$f_{0}=1, f_{1}=1, g_{0} \rightarrow 0, g_{1} \rightarrow 0, h_{0} \rightarrow 0, h_{1} \rightarrow 0, h_{2} \rightarrow 0$ as $y \rightarrow \infty$

The Equations (18) - (23) are still coupled and non-linear, whose exact solutions are not possible. So we expand $f_{0}, f_{1}, g_{0}, g_{1}, h_{0}$ and $h_{1}$ in 
terms of Ec in the following form, as the Eckert number is very small for incompressible flows:

$$
\begin{aligned}
& f_{0}(y)=f_{01}+E c f_{02}(y) \\
& f_{1}(y)=f_{11}+E c f_{12}(y) \\
& g_{0}(y)=g_{01}+E c g_{02}(y) \\
& g_{1}(y)=g_{11}+E c g_{12}(y) \\
& h_{0}(y)=h_{01}+E_{c h}(y) \\
& h_{1}(y)=h_{11}+\operatorname{Ech}_{12}(y)
\end{aligned}
$$

Substituting Eqs. (26) in equations (18) - (23), equating the coefficients of harmonic and non-harmonic terms

The zeroth order equations are

$$
\begin{aligned}
& f_{01}^{\prime \prime}+f_{01}^{\prime}-N f_{01}=-\left[N+G r_{i} g_{01}+G m_{i} h_{01}\right] \\
& f_{02}^{\prime \prime}+f_{02}^{\prime}-N f_{02}=-\left[G r_{i} g_{02}+G m_{i} h_{02}\right] \\
& g_{01}^{\prime \prime}+\operatorname{Pr} g_{01}^{\prime}-R^{2} g_{01}=0 \\
& g_{02}^{\prime \prime}+\operatorname{Pr} g_{02}^{\prime}-R^{2} g_{01}+\operatorname{Pr} f_{01}^{\prime 2}=0 \\
& h_{01}^{\prime \prime}+S c h_{01}^{\prime}-K r S c h_{01}=0 \\
& h_{02}^{\prime \prime}+S c h_{02}^{\prime}-K r S c h_{02}=0
\end{aligned}
$$

The respective boundary conditions are

$$
\begin{aligned}
& f_{01}=U_{p}, f_{02}=0, g_{01}=1, g_{02}=0, h_{01}=1, h_{02}=0 \text { at } y=0 \\
& f_{01} \rightarrow 1, f_{02} \rightarrow 0, g_{01} \rightarrow 0, g_{02} \rightarrow 0, h_{01} \rightarrow 0, h_{02} \rightarrow 0 \text { as } y \rightarrow \infty
\end{aligned}
$$

The first order equations are

$$
\begin{aligned}
& f_{11}^{\prime \prime}+f_{11}^{\prime}-[N+n] f_{11}=-\left[(N+n)+G r_{i} g_{11}+G m_{i} h_{11}+A f_{01}^{\prime}\right] \\
& f_{12}^{\prime \prime}+f_{12}^{\prime}-[N+n] f_{12}=-\left[G r_{i} g_{12}+G m_{i} h_{12}+A f_{02}^{\prime}\right] \\
& g_{11}^{\prime \prime}+\operatorname{Pr} g_{11}^{\prime}-N_{1} g_{11}+A \operatorname{Pr} g_{01}^{\prime}=0 \\
& g_{12}^{\prime \prime}+\operatorname{Pr} g_{12}^{\prime}-N_{1} g_{12}+\operatorname{Pr} A g_{02}^{\prime}+2 \operatorname{Pr} f_{01}^{\prime} f_{11}^{\prime}=0 \\
& h_{11}^{\prime \prime}+S c h_{11}^{\prime}-[n+K r] S c h_{11}+A S c h_{01}^{\prime}=0 \\
& h_{12}^{\prime \prime}+S c h_{12}^{\prime}-[n+K r] S c h_{12}+A S c h_{02}^{\prime}=0
\end{aligned}
$$

The respective boundary conditions are

$$
\begin{aligned}
& f_{11}=0, f_{12}=0, g_{11}=1, g_{12}=0, h_{11}=1, h_{12}=0 \quad \text { at } y=0 \\
& f_{11} \rightarrow 1, f_{12} \rightarrow 0, g_{11} \rightarrow 0, g_{12} \rightarrow 0, h_{11} \rightarrow 0, h_{12} \rightarrow 0 \quad \text { as } y \rightarrow \infty
\end{aligned}
$$

Where $N_{1}=\left[n \operatorname{Pr}+R^{2}\right]$

Using above equations, we can obtain the following solutions

$$
\begin{aligned}
& f=f_{0}+\varepsilon e^{n t} f_{1} \\
& =\left[f_{01}+E_{c} f_{02}\right]+\varepsilon e^{n t}\left[f_{11}+E c f_{12}\right] \\
& =\left[\begin{array}{l}
\left(F_{6} e^{-r_{5} y}+1-F_{4} e^{-r_{3} y}-F_{5} e^{-r_{1} y}\right)+ \\
E c\left(\begin{array}{l}
F_{21} e^{-r_{7} y}-F_{14} e^{-r_{6} y}+F_{15} e^{-2 r_{5} y}+F_{16} e^{-2 r_{3} y} \\
+F_{17} e^{-2 r_{1} y}-F_{18} e^{-\left(r_{5}+r_{3}\right) y}+F_{19} e^{-\left(r_{3}+r_{1}\right) y}-F_{20} e^{-\left(r_{5}+r_{1}\right) y}
\end{array}\right)
\end{array}\right) \\
& +\varepsilon e^{n t}\left[\begin{array}{l}
\left(1+F_{27} e^{-r_{1} y}-F_{22} e^{-r_{4} y}-F_{23} e^{-r_{3} y}-F_{24} e^{-r_{2} y}-F_{25} e^{-r_{1} y}+F_{26} e^{-r_{5} y}\right)+ \\
E c\left(\begin{array}{l}
F_{63} e^{-r_{10} y}-F_{45} e^{-r_{9} y}-F_{46} e^{-r_{6} y}+F_{47} e^{-2 r_{5} y}+F_{48} e^{-2 r_{3} y}+ \\
F_{49} e^{-2 r_{1} y}-F_{50} e^{-\left(r_{5}+r_{3}\right) y}+F_{51} e^{-\left(r_{3}+r_{1}\right) y}-F_{52} e^{-\left(r_{5}+r_{1}\right) y}+ \\
F_{53} e^{-\left(r_{5}+r_{8}\right) y}-F_{54} e^{-\left(r_{5}+r_{4}\right) y}-F_{55} e^{-\left(r_{5}+r_{2}\right) y}-F_{56} e^{-\left(r_{8}+r_{3}\right) y}+ \\
F_{57} e^{-\left(r_{3}+r_{4}\right) y}+F_{58} e^{-\left(r_{3}+r_{2}\right) y}-F_{59} e^{-\left(r_{1}+r_{8}\right) y}+F_{60} e^{-\left(r_{8}+r_{1}\right) y}+ \\
F_{61} e^{-\left(r_{2}+r_{1}\right) y}+F_{62} e^{-r_{7} y}
\end{array}\right)
\end{array}\right]
\end{aligned}
$$

$$
\begin{aligned}
g & =g_{0}+\varepsilon e^{n t} g_{1} \\
& =\left[g_{01}+E c g_{02}\right]+\varepsilon e^{n t}\left[g_{11}+E c g_{12}\right] \\
& =\left[\left(e^{-r_{3} y}\right)+E c\left(\begin{array}{l}
\left.F_{13} e^{-r_{6} y}-F_{7} e^{-2 r_{5} y}-F_{8} e^{-2 r_{3} y}-F_{9} e^{-2 r_{1} y}+\right) \\
F_{10} e^{-\left(r_{5}+r_{3}\right) y}-F_{11} e^{-\left(r_{1}+r_{3}\right) y}+F_{12} e^{-\left(r_{5}+r_{1}\right) y}
\end{array}\right)\right]+ \\
& \left.\varepsilon e^{n t}\left[\begin{array}{l}
F_{44} e^{-r_{9} y}+F_{28} e^{-r_{6} y}-F_{29} e^{-2 r_{5} y}- \\
F_{30} e^{-2 r_{3} y}-F_{31} e^{-2 r_{1} y}+F_{32} e^{-\left(r_{5}+r_{3}\right) y}- \\
F_{33} e^{-\left(r_{1}+r_{3}\right) y}+F_{34} e^{-\left(r_{1}+r_{5}\right) y}-F_{35} e^{-\left(r_{5}+r_{8}\right) y}+ \\
F_{36} e^{-\left(r_{5}+r_{4}\right) y}+F_{37} e^{-\left(r_{5}+r_{2}\right) y}+F_{38} e^{-\left(r_{8}+r_{3}\right) y}- \\
F_{39} e^{-\left(r_{4}+r_{3}\right) y}-F_{40} e^{-\left(r_{2}+r_{3}\right) y}+F_{401} e^{-\left(r_{1}+r_{8}\right) y}- \\
F_{42} e^{-\left(r_{1}+r_{8}\right) y}-F_{43} e^{-\left(r_{1}+r_{2}\right) y}
\end{array}\right)\right]
\end{aligned}
$$

$$
\begin{aligned}
h & =h_{0}+\varepsilon e^{n t} h_{1} \\
& =\left[e^{-r_{1} y}\right]+\varepsilon e^{n t}\left[F_{2} e^{-r_{2} y}+F_{1} e^{-r_{1} y}\right]
\end{aligned}
$$

The skin-friction coefficient, the Nusselt number and Sherwood number are important physical parameters for this type of boundary-layer flow. These parameters can be defined and determined as follows:

$$
\begin{aligned}
& C_{f}=\left.\frac{\partial f}{\partial y}\right|_{y=0} \\
& =\left[\left(-F_{6} r_{5}+F_{4} r_{3}+F_{5} r_{1}\right)+E c\left(\begin{array}{l}
F_{21} r_{7}+F_{14} r_{6}-2 F_{15} r_{5}- \\
2 F_{16} r_{3}-2 F_{17} r_{1}+F_{18}\left(r_{5}+r_{3}\right)- \\
F_{19}\left(r_{1}+r_{3}\right)+F_{20}\left(r_{5}+r_{1}\right)
\end{array}\right)\right]+ \\
& \left.\varepsilon e^{n t}\left[\begin{array}{l}
\left(-F_{27} r_{1}+F_{22} r_{4}+F_{23} r_{3}+F_{24} r_{2}+F_{25} r_{1}-F_{26} r_{5}\right)+E c \\
\left(\begin{array}{l}
-F_{63} r_{10}+F_{45} r_{9}+F_{46} r_{6}-2 F_{47} r_{5}-2 F_{48} r_{3}+ \\
2 F_{49} r_{1}+F_{50}\left(r_{5}+r_{3}\right)-F_{51}\left(r_{1}+r_{3}\right)+F_{52}\left(r_{5}+r_{1}\right)- \\
F_{53}\left(r_{5}+r_{8}\right)+F_{54}\left(r_{5}+r_{4}\right)+F_{55}\left(r_{5}+r_{2}\right)+F_{56}\left(r_{8}+r_{3}\right)- \\
F_{57}\left(r_{4}+r_{3}\right)-F_{58}\left(r_{2}+r_{3}\right)+F_{59}\left(r_{1}+r_{8}\right)-F_{60}\left(r_{1}+r_{8}\right)- \\
F_{61}\left(r_{1}+r_{2}\right)-F_{62} r_{7}
\end{array}\right)
\end{array}\right]\right) \\
& N u=-\left.\frac{\partial g}{\partial y}\right|_{y=0} \\
& =\left[\left(-r_{3}\right)+E c\left(\begin{array}{l}
-F_{13} r_{6}+2 F_{7} r_{5}+2 F_{8} r_{3}+2 F_{9} r_{1}- \\
F_{10}\left(r_{5}+r_{3}\right)+F_{11}\left(r_{1}+r_{3}\right)-F_{12}\left(r_{5}+r_{1}\right)
\end{array}\right)\right]+ \\
& \varepsilon e^{n t}\left[\left(-F_{4} r_{4}-F_{3} r_{3}\right)+E c\left(\begin{array}{l}
-F_{44} r_{9}-F_{28} r_{6}+2 F_{29} r_{5}+2 F_{30} r_{3}+ \\
2 F_{31} r_{1}-F_{32}\left(r_{5}+r_{3}\right)+F_{33}\left(r_{1}+r_{3}\right)- \\
F_{34}\left(r_{5}+r_{1}\right)+F_{35}\left(r_{5}+r_{8}\right)-F_{36}\left(r_{5}+r_{4}\right)- \\
F_{37}\left(r_{5}+r_{2}\right)-F_{38}\left(r_{8}+r_{3}\right)+F_{39}\left(r_{4}+r_{3}\right)+ \\
F_{40}\left(r_{2}+r_{3}\right)-F_{401}\left(r_{1}+r_{8}\right)+F_{42}\left(r_{1}+r_{8}\right)+ \\
F_{43}\left(r_{1}+r_{2}\right)
\end{array}\right)\right]
\end{aligned}
$$$$
S h=-\left.\frac{\partial h}{\partial y}\right|_{y=0}=\left[r_{1}\right]+\varepsilon e^{n t}\left[r_{2} F_{2}+r_{1} F_{1}\right]
$$

\section{RESULTS AND DISCUSSIONS}

Numerical evaluation of the analytical results reported in the previous section was performed and a representative set of results is reported graphically in Figs. 2-21 with $\mathrm{Sc}=0.60, \mathrm{Kr}=0.5, \mathrm{n}=0.1, \mathrm{t}=1.0, \varepsilon=$ $0.01, \mathrm{Ec}=0.01, \mathrm{~A}=0.1, \mathrm{Pr}=0.71, \mathrm{R}=0.5, \mathrm{Gr}=2, \mathrm{Gm}=2, \mathrm{Up}=0.5$, $\mathrm{M}=1.0, \alpha=\pi / 6, \xi=\pi / 3$ and $\mathrm{K}=0.5$. These results are obtained to 
illustrate the influence of the thermal Grashof number $\mathrm{Gr}$, the solutal Grashof number Gm, Prandtl number Pr, Schmidt number Sc, the plate velocity $\mathrm{Up}$, the radiation parameter $\mathrm{R}$, inclined angle $\alpha$, Aligned angle $\xi$, Permeable parameter K, Magnetic parameter M, Chemical reaction parameter $\mathrm{Kr}$ and the viscous dissipation parameter Ec on the velocity, temperature and the concentration profiles. In order to assess the accuracy of this method, we have compared our results with accepted data for the velocity and temperature profiles for the moving vertical porous plate as computed by Kim (2000). The results of these comparisons are found to be in very good agreement.

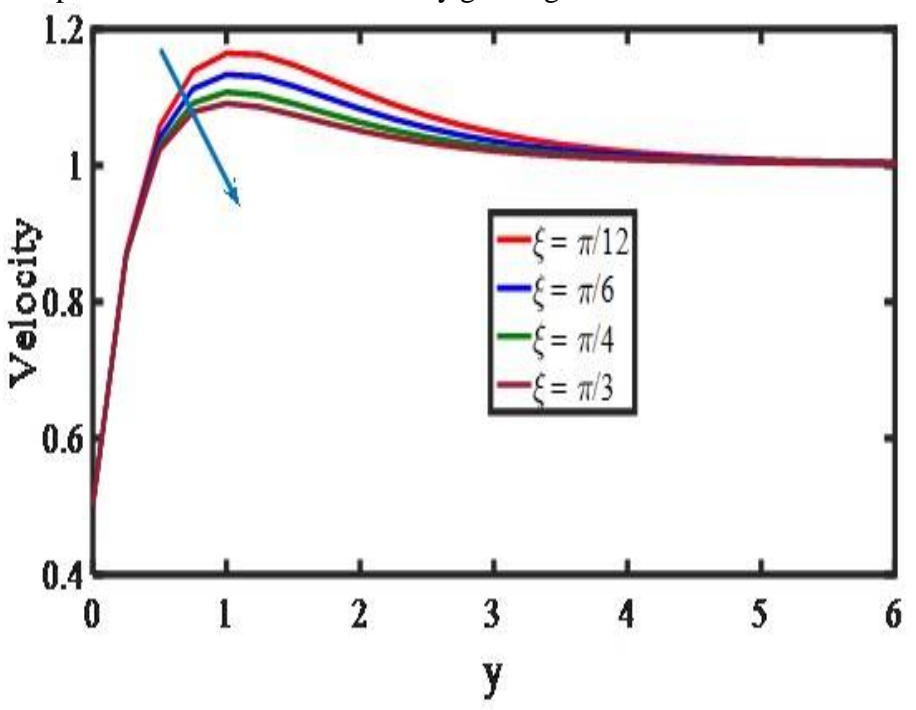

Fig. 2 Effects of $\xi$ on velocity profiles

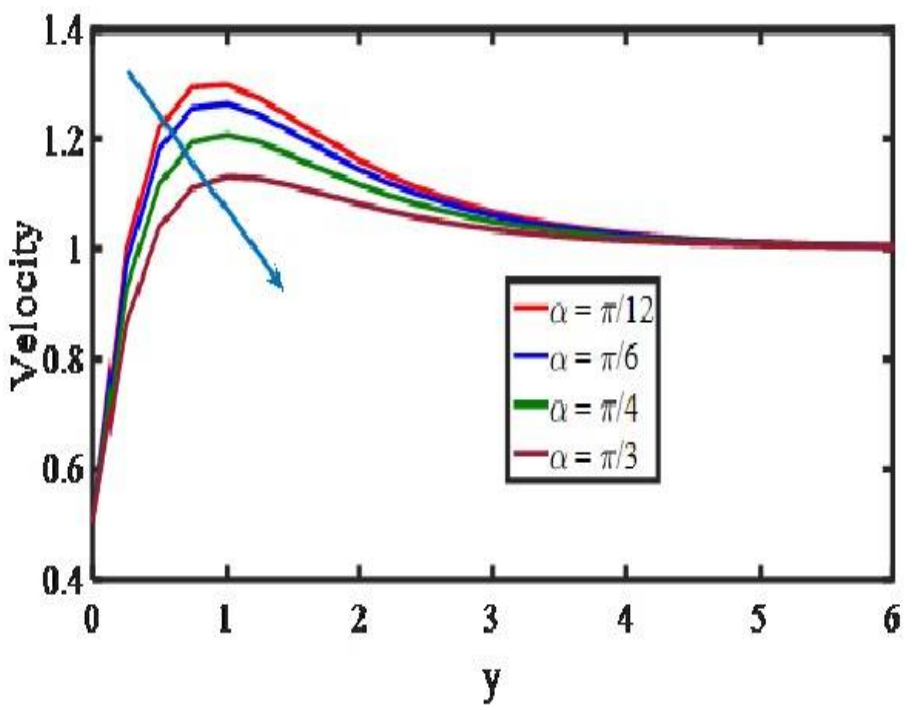

Fig. 3 Effects of $\alpha$ on velocity profiles

Figures 2and 3 demonstrate the effect of different Aligned magnetic and inclined angle values. As aligned magnetic and inclined angle increases, the velocity decreases. Fig. 8 presents the typical velocity profiles in the boundary layer for various values of the thermal Grashof number. It is observed that an increase in Gr, leads to a rise in the values of velocity due to enhancement in buoyancy force. Here, the positive values of Gr correspond to cooling of the plate. In addition, it is observed that the velocity increases rapidly near the wall of the porous plate as Grashof number increases and then decays to the free stream velocity. For the case of different values of the solutal Grashof number, the velocity profiles in the boundary layer are shown in Fig.9.The velocity distribution attains a distinctive maximum value in the vicinity of the plate and then decreases properly to approach a free stream value. As expected, the fluid velocity increases and the peak value becomes more distinctive due to increase in the buoyancy force represented by $\mathrm{Gm}$. For different values of the radiation parameter R, the velocity and temperature profiles are plotted in Figs.10 and 13. It is noticed that an increase in the radiation parameter results a decrease in the velocity and temperature within the boundary layer, as well as decreased the thickness of the velocity and temperature boundary layers. Figs. 12, 15 and 19 display the effects of Schmidt number on the velocity, temperature and concentration respectively. As the Schmidt number increases, the temperature and concentration decreases. This causes the

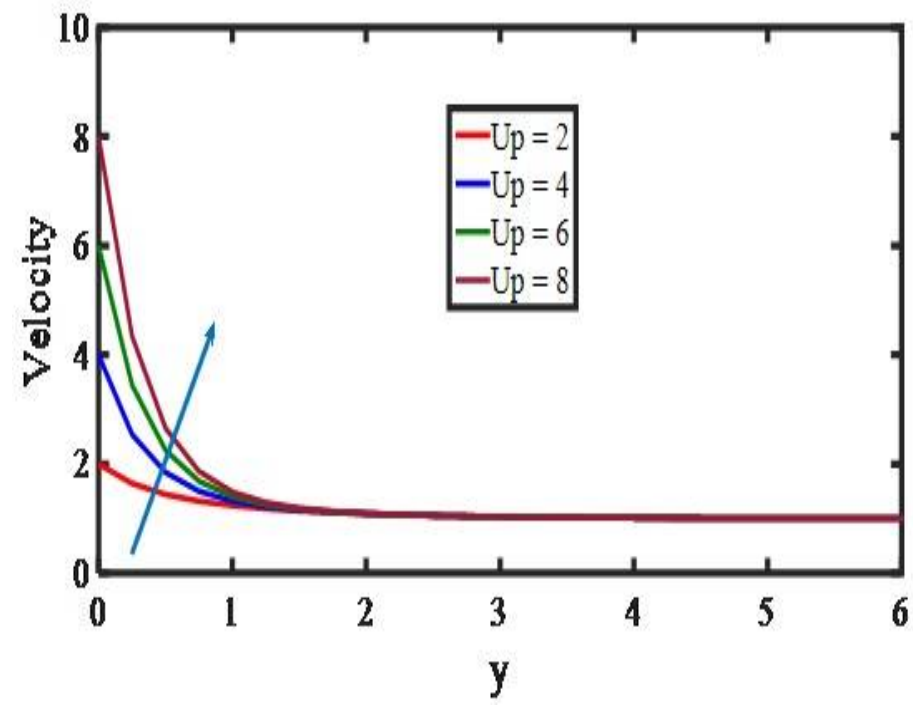

Fig. 4 Effects of Up on velocity profiles

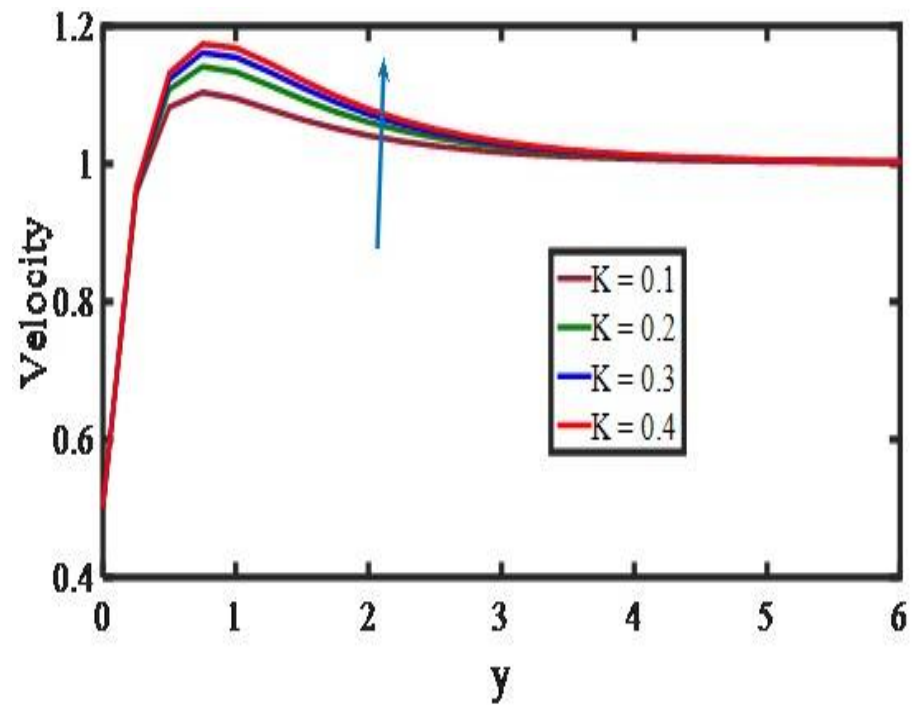

Fig. 5 Effects of $\mathrm{K}$ on velocity profiles

thermal and concentration buoyancy effects to decrease yielding a reduction in the fluid velocity. Reductions in the velocity, temperature and concentration distributions are accompanied by simultaneous reductions in the velocity, temperature and concentration boundary layers. The effects of the viscous dissipation parameter i.e., the Eckert number on the velocity and temperature are shown in Figs. 21 and 16. Greater viscous dissipative heat causes a rise in the temperature as well as the velocity. Figs.11 and 14 illustrate the behavior velocity and temperature for different values of Prandtl number. The numerical results show that the effect of increasing values of Prandtl number results in a decreasing velocity. From Fig.14, it is observed that an increase in the Prandtl number results a decrease of the thermal boundary layer thickness and in general lower average temperature within the boundary layer. The reason is that smaller values of Pr are 


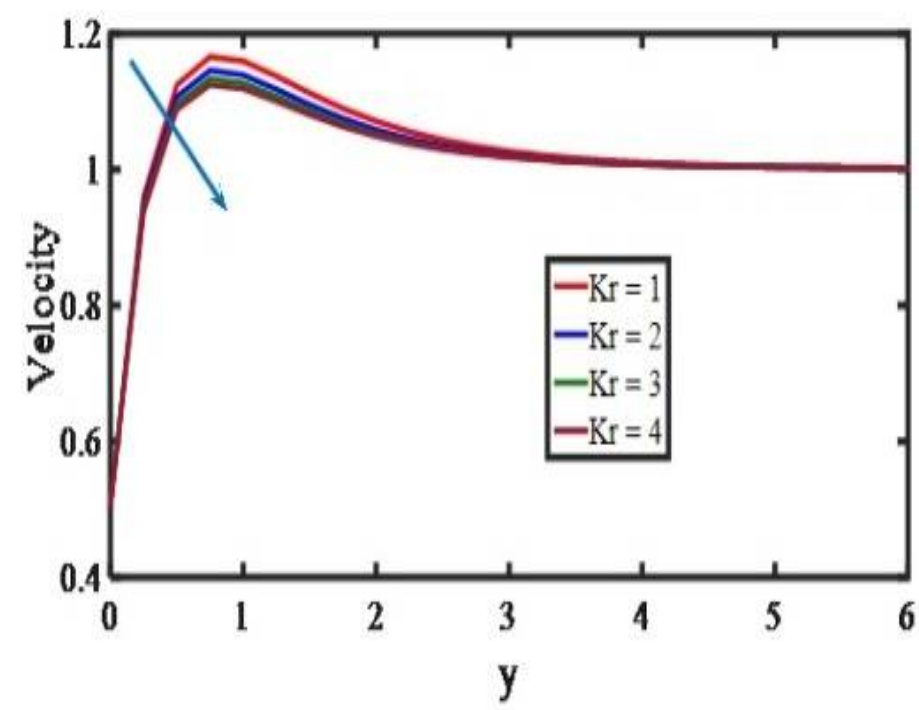

Fig. 6 Effects of $\mathrm{Kr}$ on velocity profiles

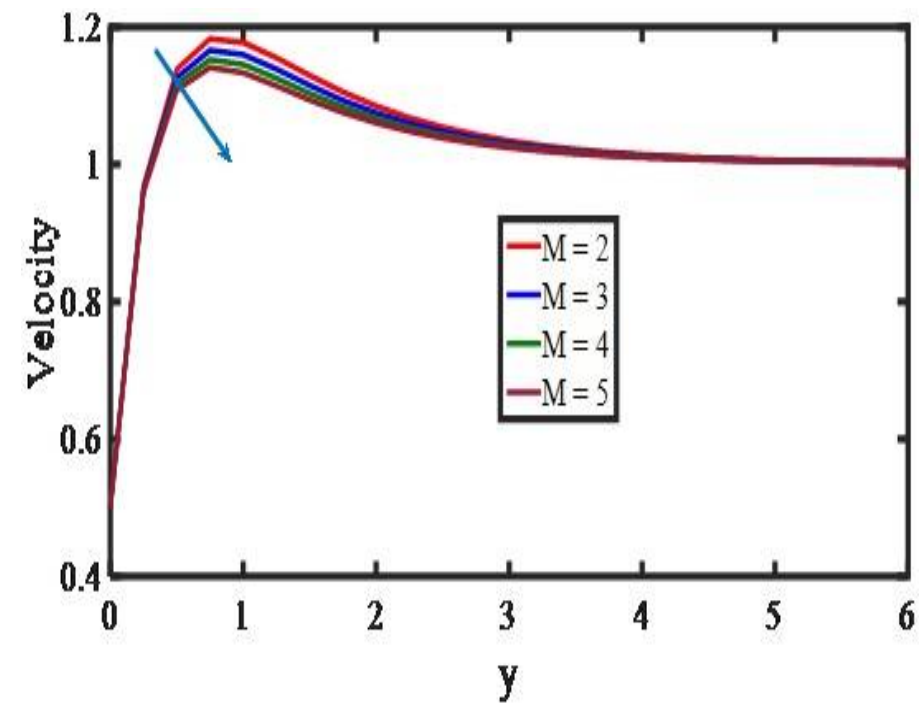

Fig. 7 Effects of $M$ on velocity profiles

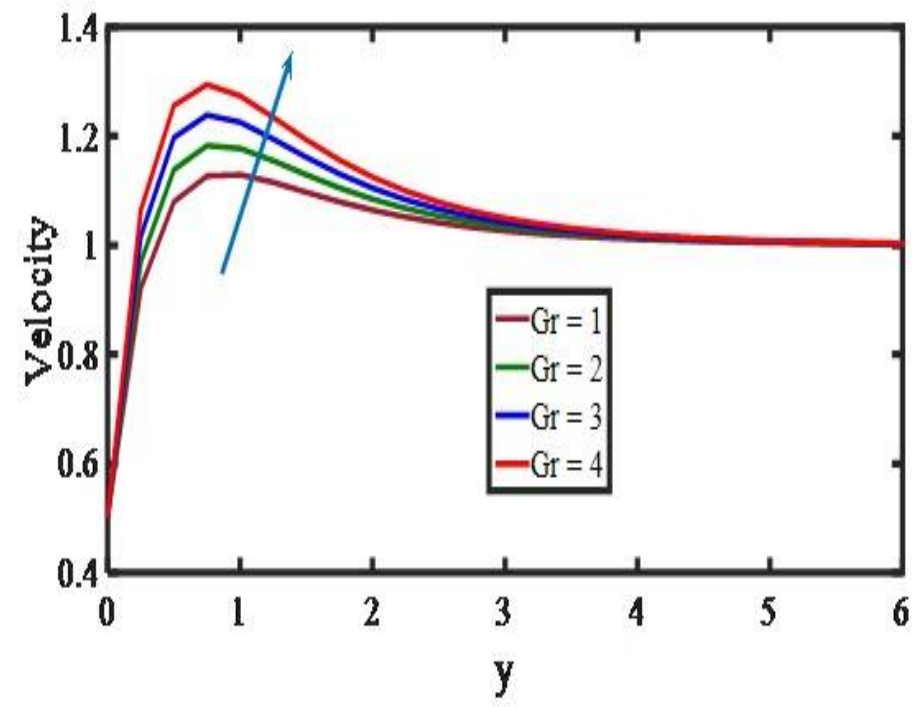

Fig. 8 Effects of Gr on velocity profiles

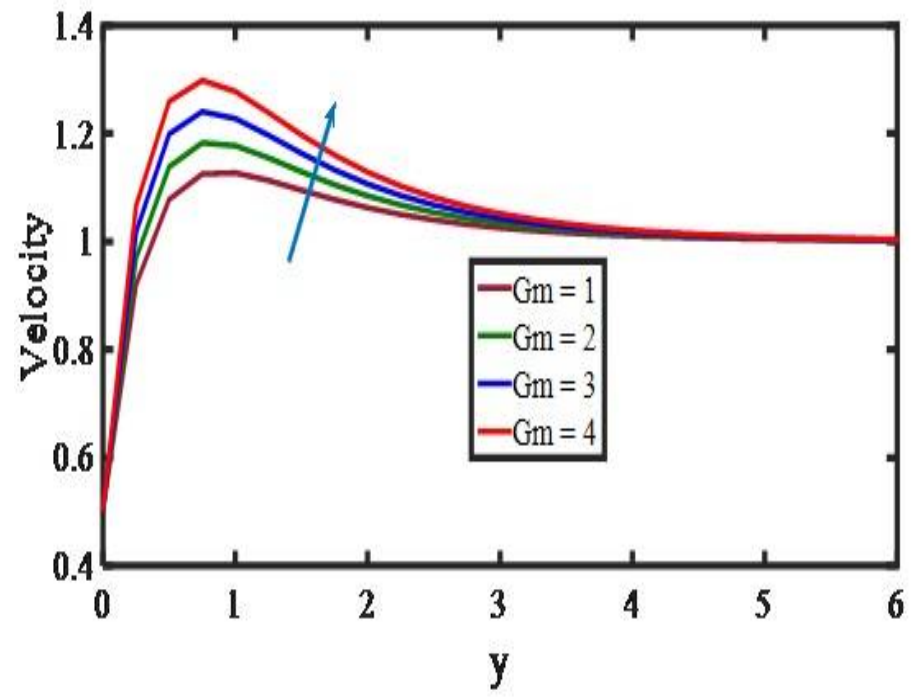

Fig. 9 Effects of Gm on velocity profiles

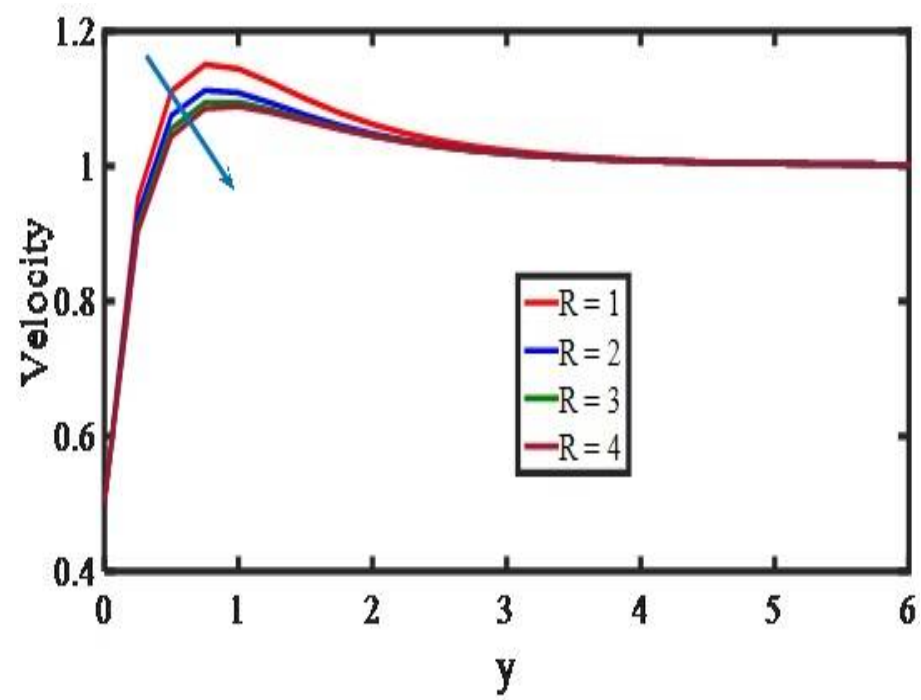

Fig. 10 Effects of $R$ on velocity profiles

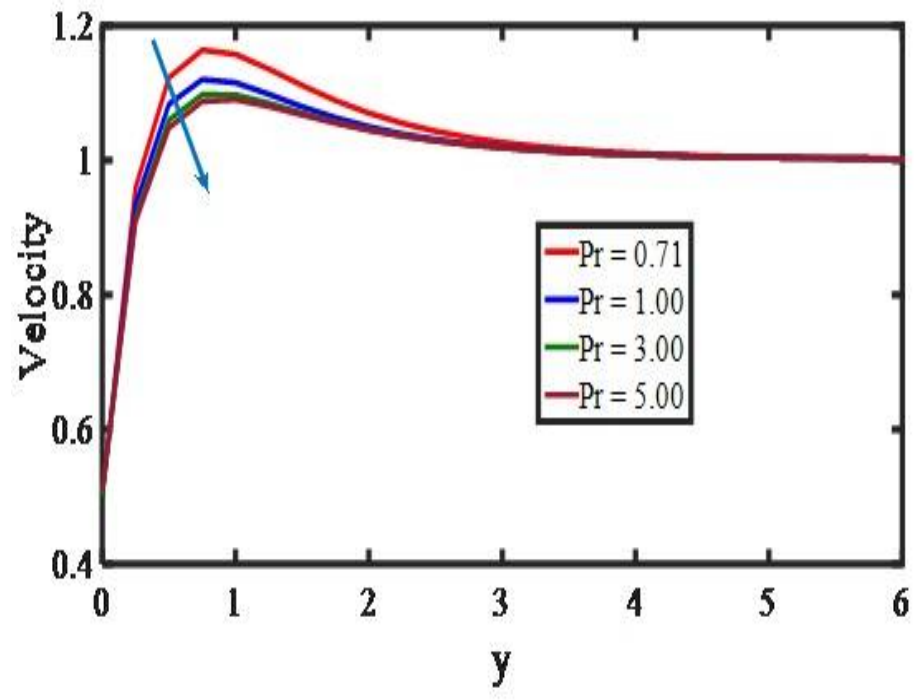

Fig. 11 Effects of Pr on velocity profiles 


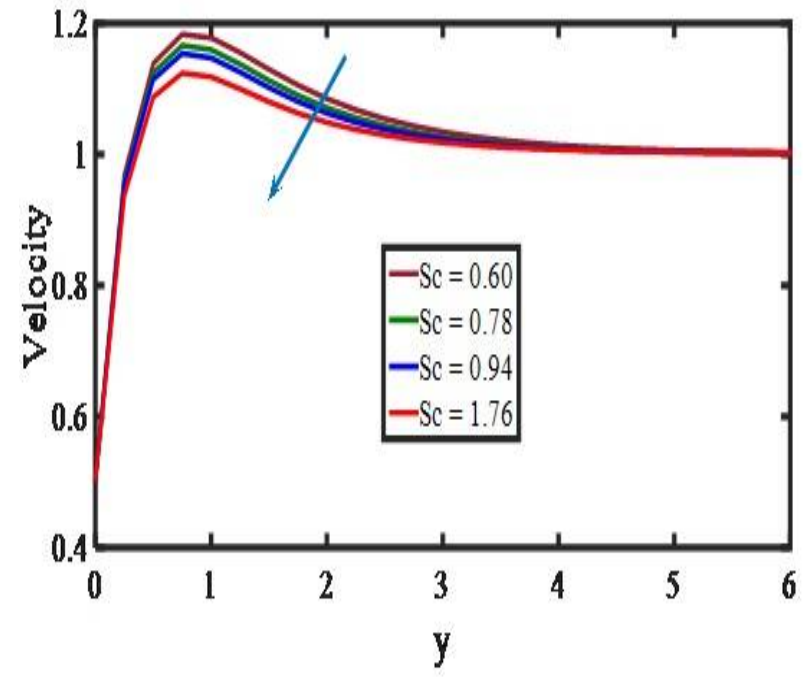

Fig. 12 Effects of Sc on velocity profiles

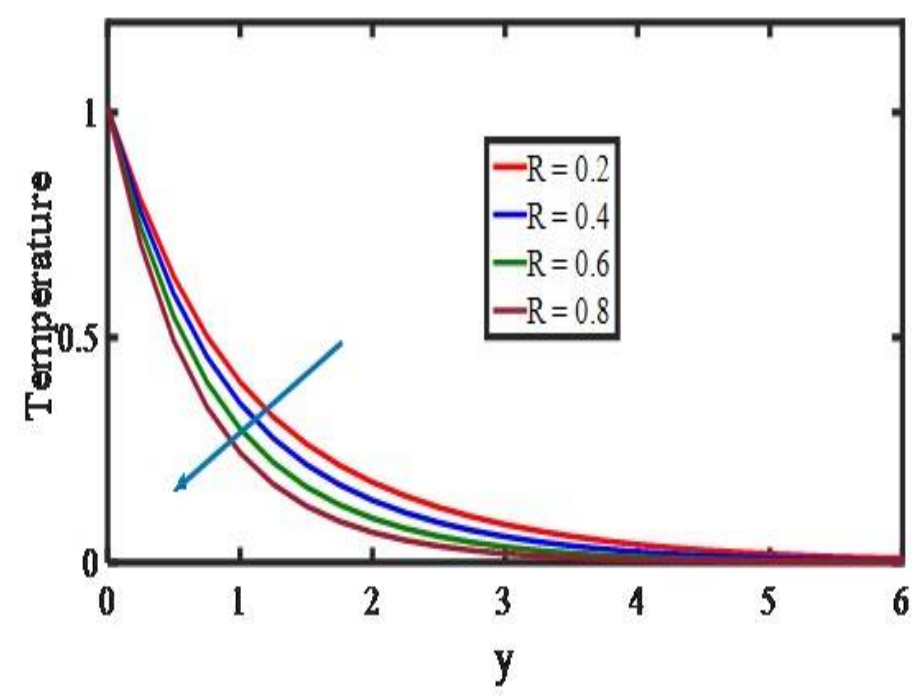

Fig. 13 Effects of $R$ on temperature profiles



Fig. 14 Effects of Pr on temperature profiles



Fig. 15 Effects of Sc on temperature profiles

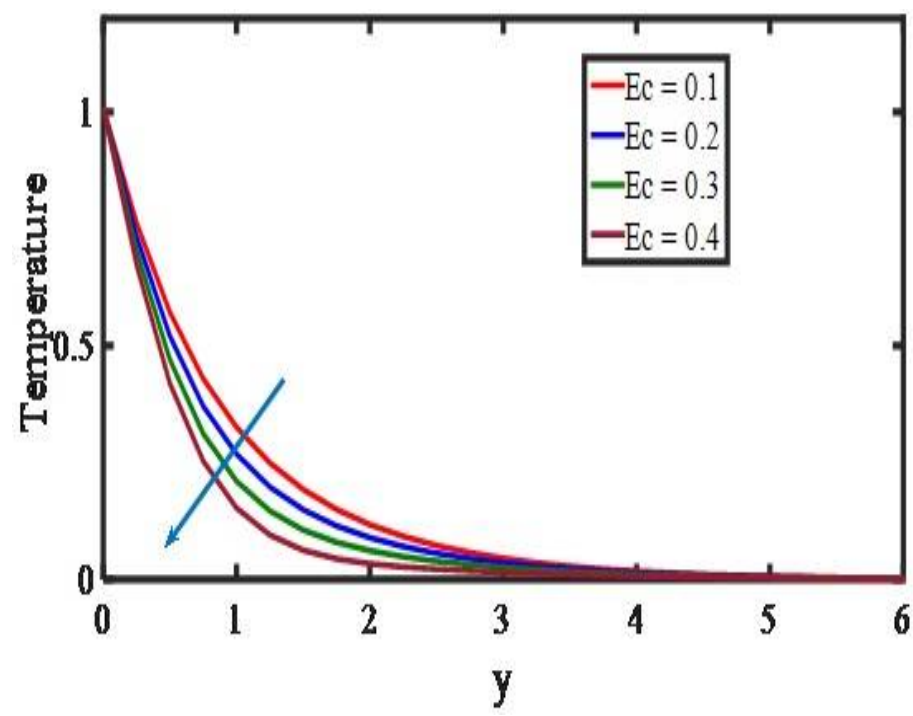

Fig. 16 Effects of Ec on temperature profiles

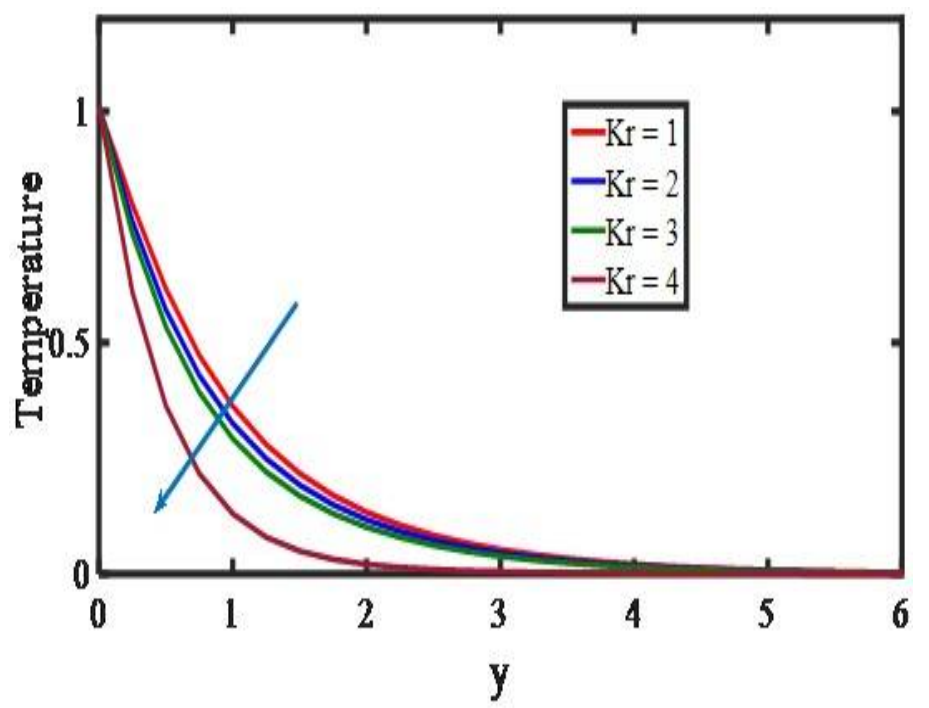

Fig. 17 Effects of $\mathrm{Kr}$ on temperature profiles 


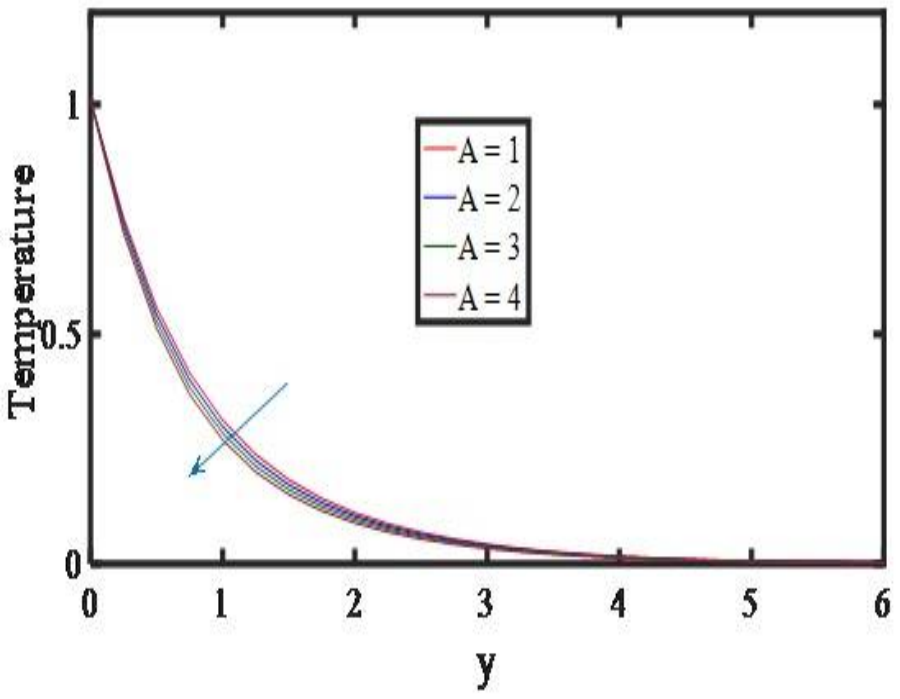

Fig. 18 Effects of A on temperature profiles

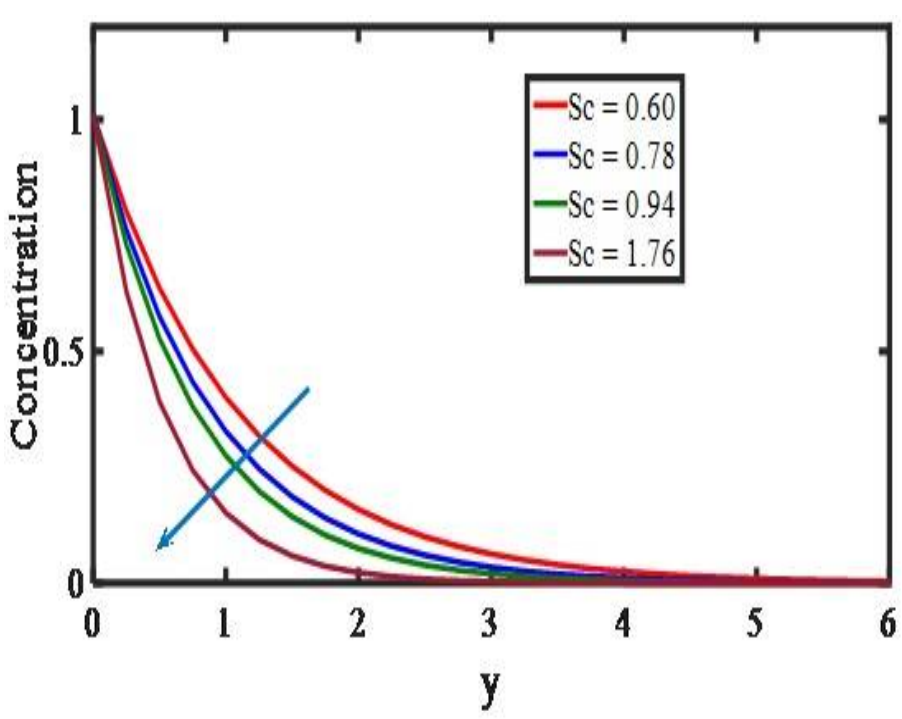

Fig. 19 Effects of Sc on concentration profiles

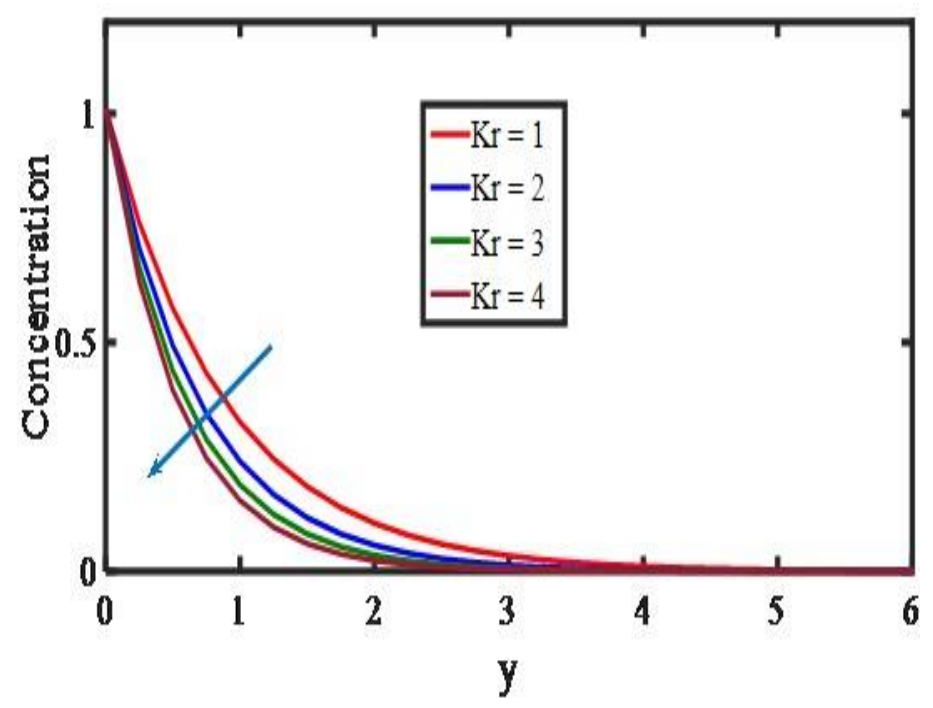

Fig. 20 Effects of $\mathrm{Kr}$ on concentration profiles

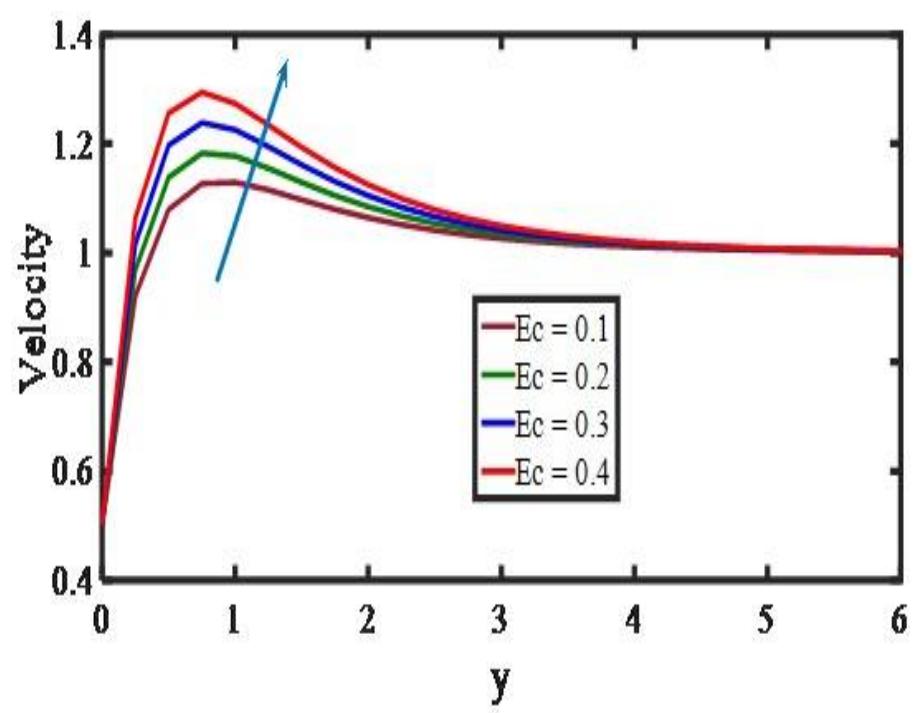

Fig. 21 Effects of Ec on velocity profiles

equivalent to increase in the thermal conductivity of the fluid and therefore heat is able to diffuse away from the heated surface more rapidly for higher values of Pr. Hence in the case of smaller Prandtl numbers as the thermal boundary layer is thicker and the rate of heat reductions in the velocity, temperature and concentration boundary layers. The effects of the viscous dissipation parameter i.e., the Eckert number on the velocity and temperature are shown in Figs. 21 and 16. Greater viscous dissipative heat causes a rise in the temperature as well as the velocity. Figs. 11 and 14 illustrate the behavior velocity and temperature for different values of Prandtl number. The numerical results show that the effect of increasing values of Prandtl number results in a decreasing velocity. From Fig.14, it is observed that an increase in the Prandtl number results a decrease of the thermal boundary layer thickness and in general lower average temperature within the boundary layer. The reason is that smaller values of $\mathrm{Pr}$ are equivalent to increase in the thermal conductivity of the fluid and therefore heat is able to diffuse away from the heated surface more rapidly for higher values of Pr. Hence in the case of smaller Prandtl numbers as the thermal boundary layer is thicker and the rate of heat transfer is reduced. The effects of the chemical reaction parameter on the velocity, temperature and concentration are shown in Figs. 6, 17 and 20 . It is noticed that an increase in the chemical reaction parameter results a decrease in the velocity, temperature and concentration within the boundary layer. For various values of the magnetic parameter $M$, the velocity profiles are plotted in Fig.7. It is obvious that existence of the magnetic field decreases the velocity. Fig. 5 shows the velocity profiles for different values of the permeability parameter. Clearly, as $K$ increases the peak values of the velocity tends to increase. Fig.4 presents the variation of the velocity distribution across the boundary layer for different values of the plate velocity Up in the direction of the fluid flow. Although we have different initial plate velocities, the velocity decreases to the constant value for given material parameters. Figure 18 depicts that temperature depreciates with the unsteady parameter A. Each and every fluid has the similar effect on temperature for the unsteady parameter A.

Tables 1-3 depict the effects of the thermal Grashof number Gr, the solutal Grashof number Gm, Prandtl number Pr, Schmidt number Sc, the plate velocity $\mathrm{Up}$, the Radiation parameter $\mathrm{R}$, inclined angle $\alpha$, Aligned angle $\xi$, Permeable parameter $\mathrm{K}$, Magnetic parameter $\mathrm{M}$ and Chemical reaction parameter $\mathrm{Kr}$ on the skin-friction coefficient $\mathrm{C}_{\mathrm{f}}$, Nusselt number $\mathrm{Nu}$ and the Sherwood number Sh, respectively. It is observed from these tables that as $\mathrm{Gr}$ increases, the skin-friction coefficient increases. However, as the radiation effects increase, the skin-friction coefficient increases and the Nusselt number decreases. Also, increases in the Schmidt number cause reductions in the skinfriction coefficient and enchance the Sherwood number. From Table 1, 
it can be seen that as the $\mathrm{Sc}, \mathrm{Kr}, \mathrm{Pr}, \mathrm{R}, \mathrm{M}, \mathrm{K}$ and Ec increases, the skinfriction decreases while increases with enchancing $\mathrm{Gr}$ and $\mathrm{Gm}$. However, from Table 2, it is noticed that, an increase $\mathrm{Kr}$, the Nusselt number decreases while the $\mathrm{Pr}, \mathrm{R}$ and $\mathrm{Sc}$ increases, Nusselt value is increases Finally, from Table 3, it is observed that as $\mathrm{Sc}, \mathrm{Kr}$ and $\mathrm{A}$ increases the skin-friction the Sherwood number increases.

Table 1 The Skin-friction for various values of Sc, Kr, Pr, R, Gr, Gm, $\mathrm{M}, \mathrm{K}$ and Ec.

\begin{tabular}{|c|c|c|c|c|c|c|c|c|c|}
\hline$S c$ & $K r$ & $P r$ & $R$ & $G r$ & $G m$ & $M$ & $K$ & $E c$ & $C f$ \\
\hline $\begin{array}{l}0.30 \\
0.60 \\
0.78 \\
0.94\end{array}$ & & & & & & & & & $\begin{array}{l}3.1112 \\
2.9319 \\
2.8426 \\
2.2046\end{array}$ \\
\hline & $\begin{array}{l}0.1 \\
0.2 \\
0.3 \\
0.4\end{array}$ & & & & & & & & $\begin{array}{l}3.0442 \\
2.0087 \\
2.9794 \\
2.9543\end{array}$ \\
\hline & & $\begin{array}{l}1 \\
3 \\
5 \\
7\end{array}$ & & & & & & & $\begin{array}{l}2.7827 \\
2.5953 \\
2.4610 \\
2.3671\end{array}$ \\
\hline & & & $\begin{array}{l}0.1 \\
0.2 \\
0.3 \\
0.4\end{array}$ & & & & & & $\begin{array}{l}3.0473 \\
3.0278 \\
2.9999 \\
2.9672\end{array}$ \\
\hline & & & & $\begin{array}{l}1 \\
3 \\
4 \\
5\end{array}$ & & & & & $\begin{array}{l}2.5061 \\
3.3479 \\
3.7544 \\
4.1514\end{array}$ \\
\hline & & & & & $\begin{array}{l}1 \\
3 \\
4 \\
5\end{array}$ & & & & $\begin{array}{l}2.4939 \\
3.3674 \\
3.8006 \\
4.2313\end{array}$ \\
\hline & & & & & & $\begin{array}{l}2 \\
3 \\
4 \\
5\end{array}$ & & & $\begin{array}{l}2.8923 \\
2.8773 \\
2.8795 \\
2.8929\end{array}$ \\
\hline & & & & & & & $\begin{array}{l}0.1 \\
0.2 \\
0.3 \\
0.4\end{array}$ & & $\begin{array}{l}3.0014 \\
2.8795 \\
2.8849 \\
2.9088\end{array}$ \\
\hline & & & & & & & & $\begin{array}{l}0.01 \\
0.02 \\
0.03 \\
0.04 \\
\end{array}$ & $\begin{array}{l}2.9319 \\
2.9035 \\
2.8751 \\
2.8466 \\
\end{array}$ \\
\hline
\end{tabular}

Table 2 The Nusselt number for various values of $\mathrm{Pr}, \mathrm{R}, \mathrm{Kr}$ and Sc.

\begin{tabular}{|c|c|c|c|c|}
\hline$P r$ & $R$ & $K r$ & $S c$ & $N u$ \\
\hline 1 & & & & 1.0917 \\
3 & & & & 2.8282 \\
5 & & & & 4.6767 \\
7 & & & & 6.5414 \\
\hline & 0.1 & & & 0.6192 \\
& 0.2 & & & 0.6609 \\
& 0.3 & & & 0.7226 \\
& 0.4 & & & 0.7975 \\
\hline & & 0.1 & & 0.9086 \\
& & 0.2 & & 0.9012 \\
& & 0.3 & & 0.8934 \\
& & 0.4 & & 0.8867 \\
\hline & & & 0.30 & 0.8656 \\
& & & 0.60 & 0.8809 \\
& & & 0.78 & 0.8867 \\
& & & 0.94 & 0.8906 \\
\hline
\end{tabular}

Table 3 The Sherwood number for various values of $\mathrm{Sc}, \mathrm{Kr}$ and $\mathrm{A}$

\begin{tabular}{|c|c|c|c|}
\hline$S c$ & $K r$ & $A$ & $S h$ \\
\hline 0.30 & & & 0.5722 \\
0.45 & & & 0.7591 \\
0.60 & & & 0.9357 \\
0.78 & & & 1.1399 \\
\hline & 0.1 & & 0.3842 \\
& 0.2 & & 0.4428 \\
& 0.3 & & 0.4915 \\
& 0.4 & & 0.5340 \\
\hline & & 1 & 0.5741 \\
& & 2 & 0.5763 \\
& & 3 & 0.5785 \\
& & 4 & 0.5806 \\
\hline
\end{tabular}

\section{CONCLUSIONS}

The governing equations for unsteady MHD convective heat and mass transfer past a semi infinite inclined permeable moving plate embedded in a porous medium with thermal radiation and viscous dissipation was formulated. The plate velocity was maintained at a constant value and the flow was subjected to a transverse magnetic field. The resulting partial differential equations were transformed into a set of ordinary differential equations using two-term series and solved in closed-form. Numerical evaluations of the closed-form results were performed and some graphical results were obtained to illustrate the details of the flow and heat and mass transfer characteristics and their dependence on some of the physical parameters. It was found that when the solutal Grashof number increased, the concentration buoyancy effects were enhanced and thus, the fluid velocity increased. However, the presence of heat radiation effects caused reductions in the fluid temperature which resulted in decreases in the fluid velocity. Also, when the Schmidt number was increased, the concentration level was decreased resulting in a decreased fluid velocity. In addition, it was found that the skin-friction coefficient increased due to decreases in the concentration buoyancy effects while it decreased due to increases in either of the heat radiation coefficient or the Schmidt number. However, the Nusselt number decreased as the heat radiation coefficient was increased and the Sherwood number decreased as the Schmidt number was increased. The temperature of the fluid decreases for increasing values of Prandtl number and viscous dissipation parameter. The existence of chemical reaction leads to decrease the concentration of the fluid. The rate of heat transfer rises for increasing values of Prandtl number and Schmidt number and decreasing values of chemical reaction parameter.

\section{NOMENCLATURE}

A

$\mathrm{B}_{0}$

c

$\mathrm{Cp}$

$C^{*}$

$\mathrm{Ec}$

$\mathrm{C}_{\mathrm{f}}$

D

Gr

$\mathrm{Gm}$

$\mathrm{g}$

K

$\mathrm{k}$

M

$\mathrm{N}$

$\mathrm{n}$

$\mathrm{Nu}$

$\operatorname{Pr}$ suction velocity parameter magnetic induction (A.m²) concentration specific heat at constant pressure $\left(\mathrm{J} \mathrm{kg}^{-1} \mathrm{~K}\right)$

dimensionless concentration $\left(\mathrm{kg} \mathrm{m}^{3}\right)$

viscous dissipation parameter or the Eckert number skin-friction coefficient mass diffusion coefficient solutal Grashof number thermal Grashof number accelration due to gravity $\left(\mathrm{ms}^{-2}\right)$ permeability of the porous medium thermal conductivity magnetic filed parameter $\left(\mathrm{Am}^{-1}\right)$ dimentionless material parameter dimensionless exponential index Nusselt number Prandtl number 


$\begin{array}{ll}\mathrm{Re}_{\mathrm{x}} & \text { local Reynolds number } \\ \mathrm{Sc} & \text { Schmidt number } \\ \mathrm{Sh} & \text { Sherwood number }\left(\mathrm{Kg} / \mathrm{m}^{2} \mathrm{~s}\right) \\ \mathrm{T} & \text { temperature } \\ \mathrm{t} & \text { dimentionless time }(\mathrm{sec}) \\ \mathrm{U}_{0} & \text { scale of free stream velocity } \\ \mathrm{u}, \mathrm{v} & \text { components of velocities along and perpendicula } \\ & \text { to the plate, respectively } \\ \mathrm{V}_{0} & \text { scale of suction velocity }(\mathrm{m} / \mathrm{s}) \\ \mathrm{x}, \mathrm{y} & \text { distances along and perpendicular to the } \\ & \text { plate,respectivily } \\ \mathrm{q}_{\mathrm{r}} & \text { Heat flux per unit area } \\ \mathrm{R} & \text { Radiation parameter }\left(\mathrm{cm}{ }^{2}\right) \\ & \\ G r e e k ~ s y m b o l s & \\ \alpha & \text { inclined angle } \\ \beta^{*} & \text { coefficient of volumetric concentration expansion } \\ \beta & \text { coefficient of volumetric thermal expansion } \\ \varepsilon & \text { scalar constant }(<<1) \\ \sigma & \text { fluid electrical conductivity } \\ \rho & \text { fluid density }\left(\mathrm{kg} \mathrm{m}^{-3}\right) \\ \mu & \text { fluid dynamic viscosity } \\ v & \left.\text { fluid kinematic viscosity (m }{ }^{2} / \mathrm{s}\right) \\ \tau & \text { friction coefficient } \\ \theta & \text { dimensionless temperature } \\ \xi & \text { Aligned angle }\end{array}$

Superscripts

- $\quad$ differentiation with respect to y

$\begin{array}{ll}\text { Subscripts } & \\ \mathrm{p} & \text { plate } \\ \mathrm{w} & \text { wall condition } \\ \infty & \text { free stream condition }\end{array}$

\section{REFERENCES}

Ajayi, T.M., Omowaye, A.J. and Animasaun, I.L., 2017, "Viscous Dissipation Effects on the Motion of Casson Fluid over an Upper Horizontal Thermally Stratified Melting Surface of a Paraboloid of Revolution: Boundary Layer Analysis," Journal of Applied Mathematics, 2017(2017). https://doi.org/10.1155/2017/1697135

Amit, P., 2017, "Unsteady Convective Boundary Layer Flow for MHD Williamson Fluid over an Inclined Porous Stretching Sheet with NonLinear Radiation and Heat Source," International Journal of Applied and Computational Mathematics, 3(1), 859-881.

https://doi.org/10.1007/s40819-017-0387-4.

Chandran, P., Sacheti,N. C., and Singh, A. K. 1998, "Unsteady Hydro magnetic Free Convection Flow with Heat Flux and Accelerated Boundary motion," Journal of the Physical Society of Japan, 67, 124 129. http://dx.doi.org/10.1143/jpsj.67.124

Georgantopoulos, G. A., Douskos, C. N., Kafousiasand, N.G., and Goudas, C. L., 1979, "Hydromagnetic Free Convection Effects on the Stokes problem for an Infinite Vertical Plate". Letters Heat and Mass Transfer, 6, 397-404. http://dx.doi.org/10.1016/0094-4548(79)90050-x

Jithender Reddy, G., Srinivasa Raju, R. and Anand Rao, J., 2017, "Influence of Viscous Dissipation on Unsteady MHD Natural
Convective Flow of Casson Fluid over an Oscillating Vertical Plate via FEM," Ain Shams Engineering Journal. http://dx.doi.org/10.1016/j.asej.2016.10.012

Monica Medikar., Sucharitha Joga and Kishore Kumar Chidem., 2016, "MHD Stagnation Point Flow of a Casson Fluid over a Nonlinearly Stretching Sheet with Viscous Dissipation," American Journal of Computational Mathematics, 6, 37-48.

http://dx.doi.org/10.4236/ajcm.2016.61005

Ogulu, A., and Makinde, O. D., 2009, “Unsteady Hydromagnetic Free Convection Flow of a Dissipative and Rotating Fluid past a Vertical Plate with Constant Heat Flux," Chemical Engineering Communication, 196, 454-462.

https://doi.org/10.1080/00986440802484531

Prabhakar Reddy, B., 2014 "Effects of Thermal Diffusion and Viscous Dissipation on Unsteady MHD Free Convection flow past a Vertical Porous Plate under Oscillatory Suction Velocity with Heat Sink". International Journal of Applied Mechanics and Engineering, 19(2), 303-320.

http://dx.doi.org/10.2478/ijame-2014-0020

Pramanik, S., 2014, "Casson Fluid Flow and Heat Transfer Past an Exponentially Porous Stretching Surface in Presence of Thermal Radiation," Ain Shams Engineering Journal, 5, 205-212. http://dx.doi.org/10.1016/j.asej.2013.05.003

Pushpalatha, K., Sugunamma, V., Ramana Reddy, J.V. and Sandeep, N., 2016, "Heat and Mass Transfer in Unsteady MHD Casson Fluid Flow with Convective Boundary Conditions," International Journal of Advanced Science and Technology, 91, 19-38. http://dx.doi.org/10.14257/ijast.2016.91.03

Raju, M.C., Ananda Reddy, N., and Varma, S.V.K., 2014, “Analytical Study of MHD Free Convictive, Dissipative Boundary Layer Flow Past a Porous Vertical Surface in the Presence of Thermal Radiation, Chemical Reaction and Constant Suction," Ain Shams Engineering Journal, 5 (4), 1361-1369. https://doi.org/10.1016/j.asej.2014.07.005.

Raptis, A., Perdikis, C., and Leontitsis, A., 2003, "Effects of Radiation in an Optically Thin Gray Gas Flowing Past a Vertical Infinite Plate in the Presence of a Magnetic Field," Heat Mass Transfer, 39, 771 - 773. https://doi.org/10.1007/s00231-002-0317-8

Rawi, N.A., Ilias, M.R., Isa, Z.M., and Shafie, S., 2016, "G-Jitter Induced Mixed Convection Flow and Heat Transfer of Micropolar Nanofluid Flow over an Inclined Stretching Sheet," International Conference on Mathematics, Engineering and Industrial Applications, $\mathbf{1 7 7 5}(1), 030020$.

http://dx.doi.org/10.1063/1.4965140.

Sandeep, N., and Kumar, M.S.J., 2016, "Heat and Mass Transfer in Nanofluid Flow over an Inclined Stretching Sheet with Volume Fraction of Dust and Nanoparticles," Journal of Applied Fluid Mechanics, 9(5), 2205-2215.

http://dx.doi.org/10.18869/acadpub.jafm.68.236.25282

Shehzad, S.A., Hayat, T., Qasim, M. and Asghar, S., 2103, "Effects of Mass Transfer on MHD Flow of Casson Fluid with Chemical Reaction and Suction," Brazilian Journal of Chemical Engineering, 30, 187 195.

http://dx.doi.org/10.1590/S0104-66322013000100020

Srinivas, S., and Muthuraj, R., 2010, "Effects of Thermal Radiation and Space Porosity on MHD Mixed Convection Flow in a Vertical Channel 
using Homotopy Analysis Method," Communications in Nonlinear Science and Numerical Simulation, 15(8), 2098-2108.

https://doi.org/10.1016/j.cnsns.2009.09.003.

Toki, C. J., and Tokis, J. N., 2007, "Exact Solutions for the Unsteady Free Convection Flows on a Porous Plate with Time-dependent Heating", ZeitschriftfürAngewandteMathematik und Mechanik, 87, 413. http://dx.doi.org/10.1002/zamm.200510291/.

Kim, Y.J., 2000, "Unsteady MHD convective heat transfer past a semiinfinite vertical porous moving plate variable suction", Int J Eng Sci, 2000, 38, 833-45.

https://doi.org/10.1016/S0020-7225(99)00063-4 